\title{
Supplemental Tables and Figure
}

\section{Supplemental Table I A. Proteins whose expression was altered in mono-cultured EBV-B cells after}

exposure to LDIR (1 hour and 24 hour time points)

\begin{tabular}{|c|c|c|c|c|c|c|c|c|}
\hline \multirow{2}{*}{ LDIR condition } & \multirow{2}{*}{ (time point) } & \multirow{2}{*}{ accession \# } & \multirow{2}{*}{ gene name } & \multirow{2}{*}{ name } & \multicolumn{2}{|c|}{ ITRAQ 1} & \multicolumn{2}{|c|}{ iTRAQ 2} \\
\hline & & & & & fold change & p-value & fold change & $\mathrm{p}$-value \\
\hline \multirow{27}{*}{$\begin{array}{l}\text { mono-cultured EBV-B cells } \\
\text { (moB-IR vs moB-cont) }\end{array}$} & (1 hour) & P62195 & PSMC5 & $26 \mathrm{~S}$ protease regulatory subunit 8 & 1.30 & 0.046 & 1.21 & 0.039 \\
\hline & & Q10588 & BST1 & ADP-ribosyl cyclase 2 & -1.26 & 0.047 & -1.33 & 0.000 \\
\hline & & Q70UQ0 & IKBIP & Isoform 4 of Inhibitor of nuclear factor kappa-B kinase-interacting protein & -1.35 & 0.004 & -1.44 & 0.003 \\
\hline & & Q16647 & PTGIS & Prostacyclin synthase & -1.64 & 0.002 & -1.22 & 0.017 \\
\hline & & P35221 & CTNNA1 & Catenin alpha-1 & -1.69 & 0.028 & -1.25 & 0.047 \\
\hline & (24 hour) & Q96AG4 & LRRC59 & Leucine-rich repeat-containing protein 59 & 1.48 & 0.000 & 1.29 & 0.030 \\
\hline & & Q14566 & MCM6 & DNA replication licensing factor MCM6 & 1.32 & 0.001 & 1.31 & 0.019 \\
\hline & & P22234 & PAICS & Multifunctional protein ADE2 & 1.31 & 0.019 & 1.43 & 0.043 \\
\hline & & Q16531 & DDB1 & DNA damage-binding protein 1 & 1.30 & 0.001 & 1.24 & 0.025 \\
\hline & & P49591 & SARS & Serine--tRNA ligase, cytoplasmic & 1.29 & 0.029 & 1.41 & 0.003 \\
\hline & & P05455 & SSB & Lupus La protein & 1.28 & 0.027 & 1.22 & 0.012 \\
\hline & & P31146 & CORO1A & Coronin-1A & 1.28 & 0.000 & 1.33 & 0.035 \\
\hline & & P22392-2 & NME2 & Isoform 3 of Nucleoside diphosphate kinase B & 1.27 & 0.022 & 1.21 & 0.042 \\
\hline & & P78527-2 & PRKDC & Isoform 2 of DNA-dependent protein kinase catalytic subunit & 1.25 & 0.000 & 1.22 & 0.003 \\
\hline & & Q8NE71 & ABCF1 & ATP-binding cassette sub-family F member 1 & 1.24 & 0.018 & 1.28 & 0.028 \\
\hline & & 000231 & PSMD11 & $26 \mathrm{~S}$ proteasome non-ATPase regulatory subunit 11 & 1.23 & 0.003 & 1.22 & 0.002 \\
\hline & & Q13451 & FKBP5 & Peptidyl-prolyl cis-trans isomerase FKBP5 & 1.23 & 0.036 & 1.21 & 0.004 \\
\hline & & P62333 & PSMC6 & $26 \mathrm{~S}$ protease regulatory subunit $10 \mathrm{~B}$ & 1.22 & 0.012 & 1.35 & 0.047 \\
\hline & & P29692-2 & EEF1D & Isoform 2 of Elongation factor 1-delta & 1.22 & 0.034 & 1.22 & 0.050 \\
\hline & & P33991 & MCM4 & DNA replication licensing factor MCM4 & 1.22 & 0.013 & 1.21 & 0.050 \\
\hline & & Q9NTK5 & OLA1 & Obg-like ATPase 1 & 1.22 & 0.041 & 1.26 & 0.047 \\
\hline & & Q13263 & TRIM28 & Transcription intermediary factor 1-beta & 1.21 & 0.021 & 1.21 & 0.017 \\
\hline & & P07910 & HNRNPC & Heterogeneous nuclear ribonucleoproteins $\mathrm{C} 1 / \mathrm{C} 2$ & -1.27 & 0.001 & -1.31 & 0.003 \\
\hline & & P01871 & IGHM & Ig mu chain $\mathrm{C}$ region & -1.29 & 0.000 & -1.21 & 0.000 \\
\hline & & Q9uJS0 & SLC25A13 & Calcium-binding mitochondrial carrier protein Aralar2 & -1.34 & 0.033 & -1.40 & 0.039 \\
\hline & & P01834 & IGKC & Ig kappa chain $\mathrm{C}$ region & -1.37 & 0.009 & -1.22 & 0.045 \\
\hline & & Q9P0I2 & EMC3 & ER membrane protein complex subunit 3 & -1.65 & 0.048 & -1.28 & 0.047 \\
\hline
\end{tabular}

Proteins that showed a fold-change of $>1.2(\mathrm{P}<0.05)$ compared to controls are shown.

Experimental replicate $=2(\mathrm{iTRAQ} 1, \mathrm{iTRAQ} 2)$ 


\section{Supplemental Table I B. Proteins whose expression was altered in mono-cultured MSC after}

exposure to LDIR (1 hour and 24 hour time points)

\begin{tabular}{|c|c|c|c|c|c|c|c|c|}
\hline \multirow{2}{*}{ LDIR condition } & \multirow{2}{*}{ (time point) } & \multirow{2}{*}{ accession \# } & \multirow{2}{*}{ gene name } & \multirow{2}{*}{ name } & \multicolumn{2}{|c|}{ iTRAQ 1} & \multicolumn{2}{|c|}{ iTRAQ 2} \\
\hline & & & & & fold change & $\mathrm{p}$-value & fold change & $\mathrm{p}$-value \\
\hline mono-cultured MSC & (1 hour ) & P04264 & KRT1 & Keratin, type II cytoskeletal 1 & 2.45 & 0.000 & 1.21 & 0.017 \\
\hline \multirow[t]{38}{*}{ (MSC-IR vs MSC-cont) } & & P35527 & KRT9 & Keratin, type I cytoskeletal 9 & 2.01 & 0.031 & 1.65 & 0.004 \\
\hline & & P01903 & HLA-DRA & HLA class II histocompatibility antigen, DR alpha chain & 1.49 & 0.049 & 1.44 & 0.001 \\
\hline & & P12270 & TPR & Nucleoprotein TPR & 1.45 & 0.044 & 1.23 & 0.042 \\
\hline & & P35555 & FBN1 & Fibrillin-1 & 1.45 & 0.008 & 1.21 & 0.037 \\
\hline & & 014950 & MYL12B & Myosin regulatory light chain $12 \mathrm{~B}$ & 1.33 & 0.021 & 1.36 & 0.006 \\
\hline & & Q8WU39 & PACAP & Plasma cell-induced resident endoplasmic reticulum protein & 1.29 & 0.034 & 1.29 & 0.025 \\
\hline & & P35579 & MYH9 & Myosin-9 & 1.27 & 0.000 & 1.26 & 0.001 \\
\hline & & P24534 & EEF1B2 & Elongation factor 1-beta & 1.27 & 0.045 & 1.34 & 0.001 \\
\hline & & P11413 & G6PD & Glucose-6-phosphate 1-dehydrogenase & 1.26 & 0.044 & 1.22 & 0.024 \\
\hline & & $\mathrm{P} 60660$ & MYL6 & Myosin light polypeptide 6 & 1.23 & 0.031 & 1.24 & 0.039 \\
\hline & & Q99715 & COL12A1 & Collagen alpha- $1(\mathrm{XII})$ chain & 1.23 & 0.012 & 1.33 & 0.002 \\
\hline & & P26641 & EEF1G & Elongation factor 1-gamma & 1.22 & 0.011 & 1.48 & 0.012 \\
\hline & & P62937 & PPIA & Peptidyl-prolyl cis-trans isomerase $\mathrm{A}$ & 1.22 & 0.011 & 1.21 & 0.004 \\
\hline & & P50990 & сст8 & T-complex protein 1 subunit theta & 1.22 & 0.001 & 1.21 & 0.005 \\
\hline & & P13693 & TPT1 & Translationally-controlled tumor protein & -1.27 & 0.044 & -1.21 & 0.000 \\
\hline & & P12111 & COL6A3 & Collagen alpha-3(VI) chain & -1.27 & 0.005 & -1.45 & 0.004 \\
\hline & & P02545 & LMNA & Prelamin-AVC & -1.28 & 0.000 & -1.21 & 0.000 \\
\hline & & P31689 & DNAJA1 & DnaJ homolog subfamily A member 1 & -1.29 & 0.046 & -1.22 & 0.005 \\
\hline & & Q96HY6 & DDRGK1 & DDRGK domain-containing protein 1 & -1.31 & 0.009 & -1.29 & 0.044 \\
\hline & & P50454 & SERPINH1 & Serpin $\mathrm{H} 1$ & -1.51 & 0.000 & -1.41 & 0.005 \\
\hline & (24 hour) & P07602 & PSAP & Proactivator polypeptide & 1.74 & 0.017 & 1.22 & 0.005 \\
\hline & & Q15293 & RCN1 & Reticulocalbin-1 & 1.37 & 0.015 & 1.39 & 0.019 \\
\hline & & P02545 & LMNA & Prelamin-AVC & 1.36 & 0.000 & 1.22 & 0.000 \\
\hline & & Q96HE7 & ERO1L & ERO1-like protein alpha & 1.35 & 0.039 & 1.56 & 0.002 \\
\hline & & P08670 & VIM & Vimentin & 1.34 & 0.000 & 1.21 & 0.033 \\
\hline & & Q9UBR2 & CTSZ & Cathepsin Z & 1.33 & 0.038 & 1.27 & 0.025 \\
\hline & & P08729 & KRT7 & Keratin, type II cytoskeletal 7 & 1.29 & 0.019 & 1.57 & 0.003 \\
\hline & & Q12797 & ASPH & Aspartyl/asparaginyl beta-hydroxylase & 1.29 & 0.017 & 1.24 & 0.048 \\
\hline & & 043707 & ACTN4 & Alpha-actinin-4 & 1.27 & 0.021 & 1.22 & 0.000 \\
\hline & & P12814 & ACTN1 & Alpha-actinin-1 & 1.25 & 0.011 & 1.21 & 0.017 \\
\hline & & P30040 & ERP29 & Endoplasmic reticulum resident protein 29 & 1.24 & 0.034 & 1.31 & 0.023 \\
\hline & & Q99623 & PHB2 & Prohibitin-2 & 1.23 & 0.009 & 1.21 & 0.042 \\
\hline & & P35222 & CTNNB1 & Catenin beta-1 & 1.22 & 0.027 & 1.23 & 0.037 \\
\hline & & P14625 & HSP90B1 & Endoplasmin & 1.22 & 0.008 & 1.22 & 0.008 \\
\hline & & P30050 & RPL12 & $60 \mathrm{~S}$ ribosomal protein L12 & 1.22 & 0.020 & 1.40 & 0.029 \\
\hline & & Q70UQ0-4 & IKBIP & Isoform 4 of Inhibitor of nuclear factor kappa-B kinase-interacting protein & 1.21 & 0.025 & 1.31 & 0.034 \\
\hline & & P39656 & DDOST & Dolichyl-diphosphooligosaccharide--protein glycosyltransferase $48 \mathrm{kDa}$ subunit & -1.34 & 0.008 & -1.51 & 0.002 \\
\hline & & $\mathrm{P} 62987$ & UBA52 & Ubiquitin-60S ribosomal protein $\mathrm{L} 40$ & -1.40 & 0.010 & -1.21 & 0.011 \\
\hline
\end{tabular}

Proteins that showed a fold-change of $>1.2(P<0.05)$ compared to controls are shown.

Experimental replicate $=2(\mathrm{iTRAQ} 1, \mathrm{iTRAQ} 2)$ 
Supplemental Table I C. Proteins whose expression was altered in EBV-B cells co-cultured with MSC after exposure to LDIR (1 hour and 24 hour time points)

\begin{tabular}{|c|c|c|c|c|c|c|c|c|}
\hline \multirow{2}{*}{ LDIR condition } & \multirow{2}{*}{ (time point) } & \multirow{2}{*}{ accession \# } & \multirow{2}{*}{ gene name } & \multirow{2}{*}{ name } & \multicolumn{2}{|c|}{ ITRAQ 1} & \multicolumn{2}{|c|}{ ITRAQ 2} \\
\hline & & & & & fold change & $\mathrm{p}$-value & fold change & p-value \\
\hline EBV-B co-culture with MSC & (1 hour) & Q9ULV4 & CORO1C & Coronin-1C & 1.67 & 0.045 & 1.21 & 0.014 \\
\hline \multirow[t]{26}{*}{ (coB-IR vs coB-cont) } & & P49368 & сСТз & T-complex protein 1 subunit gamma & 1.60 & 0.029 & 1.23 & 0.077 \\
\hline & & Q16666 & IFI16 & Gamma-interferon-inducible protein 16 & 1.59 & 0.031 & 1.24 & 0.087 \\
\hline & & P08133 & ATP5L & ATP synthase subunit g, mitochondrial & 1.34 & 0.013 & 1.61 & 0.042 \\
\hline & & P60660-2 & MYL6 & Isoform Smooth muscle of Myosin light polypeptide 6 & 1.32 & 0.000 & 1.34 & 0.026 \\
\hline & & P60709 & VDAC1 & Voltage-dependent anion-selective channel protein 1 & 1.32 & 0.036 & 1.44 & 0.006 \\
\hline & & P01860 & IGHG3 & Ig gamma- 3 chain $\mathrm{C}$ region & 1.31 & 0.015 & 1.52 & 0.006 \\
\hline & & Q9GZT3 & SLIRP & SRA stem-loop-interacting RNA-binding protein, mitochondrial & 1.28 & 0.019 & 1.26 & 0.077 \\
\hline & & P42167 & TMPO & Lamina-associated polypeptide 2 , isoforms beta/gamma & 1.27 & 0.035 & 1.21 & 0.045 \\
\hline & & P31040 & SDHA & Succinate dehydrogenase [ubiquinone] flavoprotein subunit, mitochondrial & 1.25 & 0.049 & 1.21 & 0.075 \\
\hline & & P10809 & HSPD1 & $60 \mathrm{kDa}$ heat shock protein, mitochondrial & 1.22 & 0.039 & 1.50 & 0.006 \\
\hline & & P08670 & CYP51A1 & Lanosterol 14-alpha demethylase & -1.42 & 0.014 & -1.26 & 0.084 \\
\hline & (24 hour) & Q9HA77 & CARS2 & Probable cysteine--tRNA ligase, mitochondrial & 1.21 & 0.050 & 1.42 & 0.042 \\
\hline & & Q05655 & PRKCD & Protein kinase $\mathrm{C}$ delta type & 1.21 & 0.043 & 1.21 & 0.003 \\
\hline & & Q02543 & RPL18A & 60 S ribosomal protein L18a & -1.26 & 0.006 & -1.22 & 0.042 \\
\hline & & P19525 & EIF2AK2 & Interferon-induced, double-stranded RNA-activated protein kinase & -1.27 & 0.047 & -1.41 & 0.002 \\
\hline & & $\mathrm{P} 62241$ & RPS8 & $40 \mathrm{~S}$ ribosomal protein $\mathrm{S} 8$ & -1.27 & 0.008 & -1.23 & 0.033 \\
\hline & & P08670 & VIM & Vimentin & -1.27 & 0.000 & -1.21 & 0.014 \\
\hline & & P16401 & HIST1H1B & Histone $\mathrm{H} 1.5$ & -1.28 & 0.045 & -1.31 & 0.014 \\
\hline & & P18621 & RPL17 & 60 S ribosomal protein L17 & -1.28 & 0.018 & -1.21 & 0.004 \\
\hline & & P62888 & RPL30 & $60 \mathrm{~S}$ ribosomal protein L30 & -1.30 & 0.002 & -1.56 & 0.004 \\
\hline & & P39023 & RPL3 & $60 \mathrm{~S}$ ribosomal protein L3 & -1.31 & 0.000 & -1.41 & 0.013 \\
\hline & & Q5JPH6 & EARS2 & Probable glutamate--tRNA ligase, mitochondrial & -1.34 & 0.035 & -1.21 & 0.038 \\
\hline & & 043390 & HNRNPR & Heterogeneous nuclear ribonucleoprotein $\mathrm{R}$ & -1.37 & 0.008 & -1.29 & 0.048 \\
\hline & & $\mathrm{P} 61313$ & RPL15 & $60 \mathrm{~S}$ ribosomal protein L15 & -1.41 & 0.004 & -1.25 & 0.044 \\
\hline & & P26196 & DDX6 & Probable ATP-dependent RNA helicase DDX6 & -1.51 & 0.034 & -1.58 & 0.004 \\
\hline & & Q9BQ52 & ELAC2 & Zinc phosphodiesterase ELAC protein 2 & -1.52 & 0.041 & -1.49 & 0.047 \\
\hline bystander EBV-B of LDIR MSC & (1 hour) & Q9ULV4 & CORO1C & Coronin-1C & 2.52 & 0.036 & 1.26 & 0.004 \\
\hline \multirow{38}{*}{ (coB-BS vs coB-cont) } & & P47914 & RPL29 & $60 \mathrm{~S}$ ribosomal protein L29 & 1.72 & 0.033 & 1.58 & 0.009 \\
\hline & & P04040 & CAT & Catalase & 1.72 & 0.025 & 1.45 & 0.005 \\
\hline & & P60842 & EIF4A1 & Eukaryotic initiation factor $4 \mathrm{~A}-\mathrm{I}$ & 1.65 & 0.008 & 1.38 & 0.034 \\
\hline & & P46781 & RPS9 & 40S ribosomal protein S9 & 1.63 & 0.033 & 1.89 & 0.001 \\
\hline & & Q86UE4 & MTDH & Protein LYRIC & 1.61 & 0.031 & 1.72 & 0.001 \\
\hline & & P09669 & $\operatorname{cox} 6 \mathrm{C}$ & Cytochrome $\mathrm{c}$ oxidase subunit $6 \mathrm{C}$ & 1.60 & 0.035 & 1.21 & 0.007 \\
\hline & & P51571 & SSR4 & Translocon-associated protein subunit delta & 1.55 & 0.047 & 1.57 & 0.006 \\
\hline & & P19338 & $\mathrm{NCL}$ & Nucleolin & 1.51 & 0.000 & 1.22 & 0.044 \\
\hline & & P10412 & HIST1H1E & Histone $\mathrm{H} 1.4$ & 1.49 & 0.046 & 1.21 & 0.040 \\
\hline & & P08559-4 & PDHA1 & Isoform 4 of Pyruvate dehydrogenase E1 component subunit alpha, somatic form, mitochondrial & 1.45 & 0.050 & 1.35 & 0.036 \\
\hline & & P45880 & VDAC2 & Voltage-dependent anion-selective channel protein 2 & 1.37 & 0.037 & 1.29 & 0.001 \\
\hline & & P21333 & FLNA & $\begin{array}{l}\text { Filamin-A } \\
\text { A }\end{array}$ & 1.31 & 0.025 & 1.21 & 0.047 \\
\hline & & P21796 & VDAC1 & Voltage-dependent anion-selective channel protein 1 & 1.31 & 0.003 & 1.42 & 0.031 \\
\hline & & 075964 & ATP5L & ATP synthase subunit $\mathrm{g}$, mitochondrial & 1.29 & 0.043 & 1.22 & 0.043 \\
\hline & & P62328 & TMSB4X & Thymosin beta-4 & 1.28 & 0.029 & 1.51 & 0.007 \\
\hline & & P07437 & TUBB & Tubulin beta chain & 1.26 & 0.015 & 1.21 & 0.034 \\
\hline & & 000567 & NOP56 & Nucleolar protein 56 & 1.20 & 0.050 & 1.28 & 0.037 \\
\hline & & P31930 & UQCRC1 & Cytochrome b-c1 complex subunit 1 , mitochondrial & -1.49 & 0.034 & -1.31 & 0.048 \\
\hline & & P63244 & GNB2L1 & Guanine nucleotide-binding protein subunit beta-2-like 1 & -1.55 & 0.006 & -1.61 & 0.008 \\
\hline & & P23284 & PPIB & Peptidyl-prolyl cis-trans isomerase B & -1.77 & 0.002 & -1.23 & 0.041 \\
\hline & & Q00325-2 & SLC25A3 & Isoform B of Phosphate carrier protein, mitochondrial & -1.82 & 0.013 & -1.21 & 0.042 \\
\hline & & P52566 & ARHGDIB & Rho GDP-dissociation inhibitor 2 & -1.93 & 0.016 & -1.28 & 0.038 \\
\hline & & Q9UII2 & ATPIF1 & ATPase inhibitor, mitochondrial & -2.01 & 0.046 & -1.81 & 0.026 \\
\hline & (24 hour) & Q9NYY8 & FASTKD2 & FAST kinase domain-containing protein 2 & 1.49 & 0.040 & 1.21 & 0.035 \\
\hline & & P02765 & AHSG & Alpha-2-HS-glycoprotein & 1.43 & 0.031 & 1.31 & 0.015 \\
\hline & & Q9BTV4 & TMEM43 & Transmembrane protein 43 & 1.36 & 0.026 & 1.28 & 0.011 \\
\hline & & Q8N183 & NDUFAF2 & Mimitin, mitochondrial & 1.32 & 0.029 & 1.21 & 0.041 \\
\hline & & 000299 & CLIC1 & Chloride intracellular channel protein 1 & 1.24 & 0.037 & 1.22 & 0.030 \\
\hline & & Q02978 & SLC25A11 & Mitochondrial 2-oxoglutarate/malate carrier protein & 1.24 & 0.009 & 1.22 & 0.031 \\
\hline & & Q99442 & SEC62 & Translocation protein SEC62 & 1.24 & 0.007 & 1.31 & 0.022 \\
\hline & & Q15005 & SPCS2 & Signal peptidase complex subunit 2 & 1.23 & 0.005 & 1.29 & 0.022 \\
\hline & & P25398 & RPS12 & 40 S ribosomal protein S12 & 1.21 & 0.022 & 1.25 & 0.029 \\
\hline & & P62333 & PSMC6 & $26 \mathrm{~S}$ protease regulatory subunit $10 \mathrm{~B}$ & -1.27 & 0.011 & -1.68 & 0.001 \\
\hline & & Q96DV4 & MRPL38 & 39 S ribosomal protein L38, mitochondrial & -1.28 & 0.028 & -1.26 & 0.010 \\
\hline & & $\mathrm{P} 61313$ & RPL15 & 60 S ribosomal protein L15 & -1.29 & 0.007 & -1.39 & 0.007 \\
\hline & & Q13043 & STK4 & Serine/threonine-protein kinase 4 & -1.35 & 0.011 & -1.21 & 0.031 \\
\hline & & Q00839 & HNRNPU & Heterogeneous nuclear ribonucleoprotein $\mathrm{U}$ & -1.59 & 0.022 & -1.27 & 0.015 \\
\hline & & Q92522 & H1FX & Histone $\mathrm{H} 1 \mathrm{x}$ & -1.70 & 0.028 & -1.81 & 0.000 \\
\hline
\end{tabular}

Proteins that showed a fold-change of $>1.2(P<0.05)$ compared to controls are shown.

Experimental replicate $=2(\mathrm{iTRAQ} 1, \mathrm{iTRAQ} 2)$ 


\section{Supplemental Table II. List of genes whose expression was altered in different LDIR conditions}

detected by microarray

\begin{tabular}{lcll}
\hline \multicolumn{1}{c}{ LDIR condition } & gene name & name & fold change \\
\hline mono-cultured EBV-B cells & GPR116 & G protein-coupled receptor 116 & 1.70 \\
(moB-IR vs moB-cont) & AK4 & adenylate kinase 4 & -1.61 \\
& LRRFIP1 & leucine rich repeat (in FLII) interacting protein 1 & -1.66 \\
& PFKFB4 & 6-phosphofructo-2-kinase/fructose-2,6-biphosphatase 4 & -1.71 \\
& AREG & amphiregulin & -1.72 \\
& NR4A2 & nuclear receptor subfamily 4, group A, member 2 & -1.72 \\
& ALDOC & aldolase C, fructose-bisphosphate & -1.73 \\
& GPAM & glycerol-3-phosphate acyltransferase, mitochondrial & -1.90 \\
EBV-B co-culture with MSC & FMN1 & formin 1 & -2.06 \\
(coB-IR vs coB-cont) & TMEM229A & transmembrane protein 229A & 1.86 \\
& ND6 & NADH dehydrogenase, subunit 6 (complex I) & 1.78 \\
& OR2H2 & olfactory receptor, family 2, subfamily H, member 2 & 1.63 \\
& SULF1 & sulfatase 1 & -1.62 \\
& RNF185 & ring finger protein 185 & -1.65 \\
bystander EBV-B of LDIR MSC & SERPINB2 & serpin peptidase inhibitor, clade B (ovalbumin), member 2 & -1.68 \\
(coB-BS vs coB-cont) & & &
\end{tabular}

Genes that showed a fold-change of $>1.6$ compared to controls are shown 
Supplemental Table III. Genes predicted to be commonly targeted by let-7a, miR-16, miR-19b, and miR-21

\begin{tabular}{|c|}
\hline gene name \\
\hline CPEB3 \\
\hline ITSN1 \\
\hline TAB2 \\
\hline BSDC1 \\
\hline MAST4 \\
\hline AUH \\
\hline NLK \\
\hline TIMM23 \\
\hline ABCC5 \\
\hline SYNE1 \\
\hline CASR \\
\hline ZFYVE16 \\
\hline TGM4 \\
\hline DMD \\
\hline FNDC3B \\
\hline DTNA \\
\hline ZNF280D \\
\hline RBMS1 \\
\hline MYB \\
\hline ERGIC1 \\
\hline C1orf101 \\
\hline FAM135A \\
\hline ARMC8 \\
\hline DCUN1D3 \\
\hline KCNK1 \\
\hline ССТ6Р3 \\
\hline DNAJA2 \\
\hline MBNL1 \\
\hline ANK3 \\
\hline PHTF2 \\
\hline TRIM2 \\
\hline UBR3 \\
\hline NIPBL \\
\hline PRPF39 \\
\hline DISC1 \\
\hline HAS2 \\
\hline EIF2C3 \\
\hline LOC100272228 \\
\hline TGFBR3 \\
\hline LAMP2 \\
\hline TSNAX-DISC1 \\
\hline SYT16 \\
\hline MYO5B \\
\hline UHRF2 \\
\hline TMEM57 \\
\hline PRG4 \\
\hline A2BP1 \\
\hline LOC387647 \\
\hline NPAS3 \\
\hline DIP2A \\
\hline FAM120A \\
\hline SNRNP48 \\
\hline ALPK1 \\
\hline FOXP2 \\
\hline ORC3L \\
\hline GPAM \\
\hline NOVA1 \\
\hline TMEM135 \\
\hline CRB1 \\
\hline MYH11 \\
\hline $\mathrm{ZCCHC} 3$ \\
\hline ZBTB39 \\
\hline CNTN3 \\
\hline SLC9A6 \\
\hline EIF2AK4 \\
\hline MEX3C \\
\hline DDX3X \\
\hline HECTD2 \\
\hline NAALADL2 \\
\hline AMMECR1 \\
\hline BRWD1 \\
\hline $\mathrm{IQCH}$ \\
\hline TMEM20 \\
\hline DLG2 \\
\hline UNC13C \\
\hline SLC22A10 \\
\hline NCOA4 \\
\hline EIF2C4 \\
\hline C14orf101 \\
\hline
\end{tabular}


Supplemental Figure 1

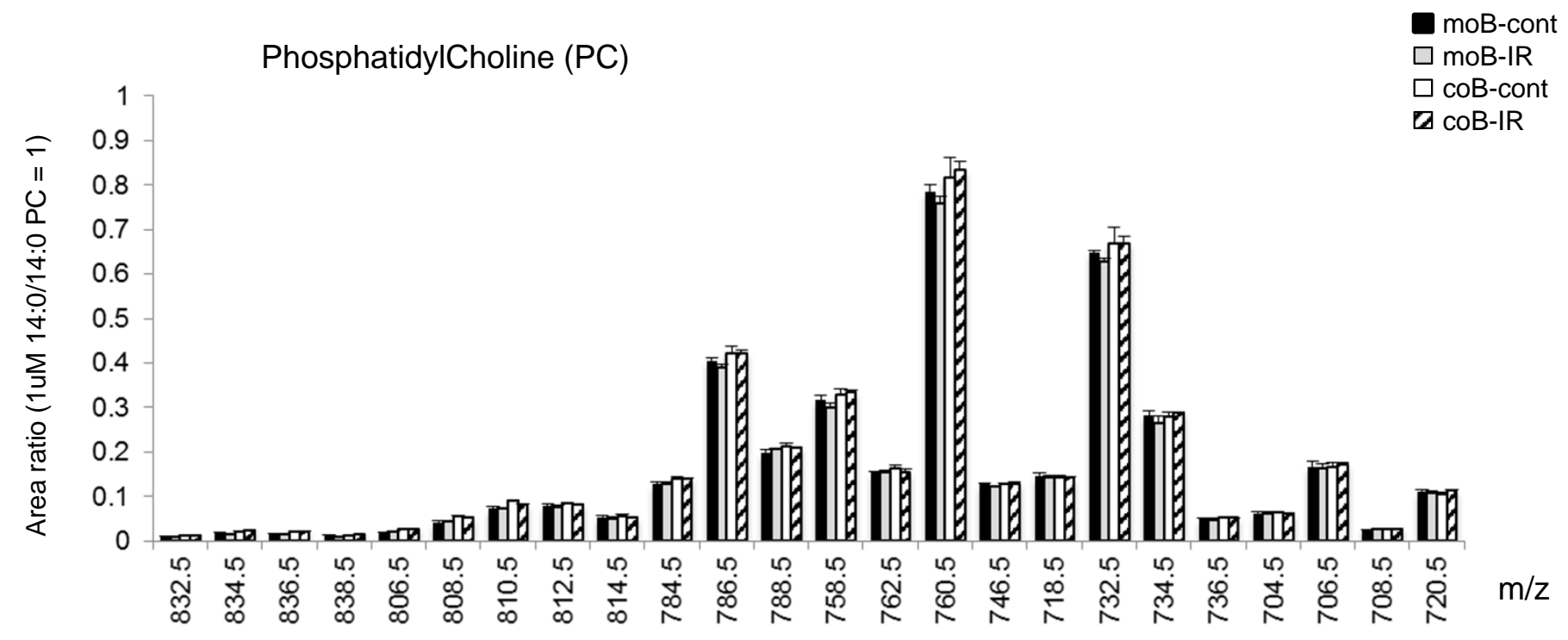

